\title{
RANDOMISATION AND RECURSION METHODS FOR MIXED-EXPONENTIAL LÉVY MODELS, WITH FINANCIAL APPLICATIONS
}

\author{
ALEKSANDAR MIJATOVIĆ, ${ }^{* * *}$ \\ MARTIJN R. PISTORIUS, ${ }^{* * * *}$ AND \\ JOHANNES STOLTE, ${ }^{* * * * *}$ Imperial College London
}

\begin{abstract}
We develop a new Monte Carlo variance reduction method to estimate the expectation of two commonly encountered path-dependent functionals: first-passage times and occupation times of sets. The method is based on a recursive approximation of the first-passage time probability and expected occupation time of sets of a Lévy bridge process that relies in part on a randomisation of the time parameter. We establish this recursion for general Lévy processes and derive its explicit form for mixed-exponential jump-diffusions, a dense subclass (in the sense of weak approximation) of Lévy processes, which includes Brownian motion with drift, Kou's double-exponential model, and hyperexponential jump-diffusion models. We present a highly accurate numerical realisation and derive error estimates. By way of illustration the method is applied to the valuation of range accruals and barrier options under exponential Lévy models and Bates-type stochastic volatility models with exponential jumps. Compared with standard Monte Carlo methods, we find that the method is significantly more efficient.
\end{abstract}

Keywords: Lévy bridge process; mixed-exponential jump-diffusion; first-passage time; occupation time; Markov bridge sampling.

2010 Mathematics Subject Classification: Primary 65C05

Secondary $91 \mathrm{G} 60$

\section{Introduction}

Motivation and brief outline. The Markov bridge sampling method for the estimation of the expectation $\mathbb{E}[F(T, \xi)]$ of a given path-functional $F$ of a Markov process $\xi$ and the horizon $T>0$ consists of averaging conditional expectations $\widetilde{F}\left(\xi_{t_{0}}, \ldots, \xi_{t_{N}}\right)$ over $M$ independent copies $\left(\xi_{t_{0}}^{(i)}, \ldots, \xi_{t_{N}}^{(i)}\right), i=1, \ldots, M$, of the values $\left(\xi_{t_{0}}, \ldots, \xi_{t_{N}}\right)$ that $\xi$ takes on the grid $\mathbb{T}_{N}=\left\{0=t_{0}<t_{1}<\cdots<t_{N}=T\right\}$ :

$$
\mathbb{E}[F(T, \xi)] \approx \frac{1}{M} \sum_{i=1}^{M} \widetilde{F}\left(\xi_{t_{0}}^{(i)}, \ldots, \xi_{t_{N}}^{(i)}\right),
$$

where $\widetilde{F}\left(\xi_{t_{0}}, \ldots, \xi_{t_{N}}\right)$ denotes the regular version of $\mathbb{E}\left[F(T, \xi) \mid \xi_{t_{0}}, \ldots, \xi_{t_{N}}\right]$. The name of the method derives from the fact that, conditional on the values $\left(\xi_{t_{0}}, \ldots, \xi_{t_{N}}\right)$, the stochastic

Received 29 May 2014; revision received 17 October 2014.

* Postal address: Department of Mathematics, Imperial College London, South Kensington Campus, London SW7 2AZ, UK.

** Email address: a.mijatovic@imperial.ac.uk

*** Email address: m.pistorius@imperial.ac.uk

**** Email address: j.stolte09@imperial.ac.uk 
processes $\left\{\xi_{t}, t \in\left[t_{i}, t_{i+1}\right]\right\}$, for $i=0, \ldots, N-1$, are equal in law to Markov bridge processes. The estimator in (1.1) is unbiased and has strictly smaller variance than the standard Monte Carlo estimator, as a consequence of the tower property of conditional expectation and the conditional variance formula. The Markov bridge sampling method has the advantage that it allows for refinements of the generated path to the required level of accuracy, and can be combined with importance sampling. Such a bridge method is especially suited to the evaluation of expectations of path-dependent functionals; see, for example, [11]. Since the function $\widetilde{F}$ is in general not available in closed or analytically tractable form, the viability of the Markov bridge method hinges on the ability to efficiently approximate the function $\widetilde{F}$. In this paper we derive an efficient approximation method for the conditional expectations $\widetilde{F}$ of certain path-dependent functionals given in terms of occupation times of sets and first-passage times, which is achieved by approximating the law of the bridge process by the law of the process pinned down at an independent random time with small variance. Since the latter law is analytically tractable when $\xi$ is a mixed-exponential Lévy process, this allows us to develop a Markov bridge Monte Carlo method for estimation of the corresponding expectation $\mathbb{E}[F(T, \xi)]$. To demonstrate the potential of the simulation method we extend the approach to a two-dimensional Markovian setting, and deploy the method to numerically approximate the values of two common path-dependent derivatives, barrier options, and range accruals, under a version of the Bates model [6], which is an example of a stochastic volatility model with jumps that is widely used in financial modelling; see [15] and [20] for background.

Literature overview. In the literature, see [19], [36], and [38], a number of bridge sampling methods exist dealing with cases in which $\xi$ is a one-dimensional Lévy process. In [19] an adaptive bridge sampling method was developed for real-valued Lévy processes based on short-time asymptotics of stopped Lévy processes. By conditioning on the jump-skeleton and exploiting the explicit form of the distribution of the maximum of a Brownian bridge, a simulation method for pricing of barrier options under jump-diffusions was presented in [36], and a refinement of this algorithm and application to the pricing of corporate bonds was given in [38]. An exact simulation algorithm for generation of diffusion sample paths deploying Brownian bridges was designed and analysed in [8]. Several alternative methods have been developed for approximation of path-dependent functionals, often based on weak or strong (pathwise) approximations of the solution of the stochastic differential equation (SDE). In the setting of diffusions, a classical treatment of various strong and weak approximation schemes was given in [28]. More recently, the problem of approximation of general path-dependent functionals has also received attention in the case of Lévy-driven SDEs. In [16] a multi-level Monte Carlo algorithm was developed for path-dependent functionals of Lévy driven SDEs that are Lipschitz continuous in the supremum norm, and identified error bounds. This algorithm is based on an approximation of the driving Lévy process by a Lévy jump-diffusion constructed by replacing the small jumps by a Brownian motion, as was investigated in [2]. Adopting an alternative approach that does not rely on the Brownian small-jump approximation, a multi-level extension was presented in [18] of the Monte Carlo method developed in [30] for estimation of Lipschitz functions of the final value and running maximum of a real-valued Lévy process. Some functionals that are of interest in various applications are not included in the analysis of [16] and [18], as these fail to satisfy the Lipschitz condition. The bridge method that we present in this paper provides approximations in two such cases; namely, the distribution of the running maximum and the expected occupation time of sets.

Approximation of bridge functionals. As mentioned above, a key step in the development of the Markov bridge method is the availability of an efficient approximation of the conditional 
expectations $\widetilde{F}$. As in general the transition probabilities of the Markov processes considered here are not explicitly available, the first step is to approximate the Markov process in question by its continuous-time Euler-Maruyama (EM) scheme. The approximation of expectations of path-dependent functionals under stochastic volatility models with jumps using the continuoustime EM scheme is based on the harness property of a Markov process which states that, for any two epochs $t_{1}$ and $t_{2}$ the collections of values of the Markov process at times in between $t_{1}$ and $t_{2}$ are independent of the values for $t$ outside this interval, conditional on the values of the process at $t_{1}$ and $t_{2}$. Noting that a Lévy process that is conditioned to start from position $x$ and to take the value $y$ at the horizon $T$ is equal in law to a Lévy bridge process from $(0, x)$ to $(T, y)$, we are led to the problem of evaluating the expectations of path-dependent functionals of Lévy bridges.

Randomisation method and recursions. The approximation method of the Lévy bridge quantities that we present is based in part on a randomisation of the time-parameter. This randomisation method was originally developed in [13] for the valuation of American put options, and is known as Erlangisation in risk theory [1, Chapter IX.8]. The method has been deployed in [3] for the efficient computation of ruin probabilities and in [4], [10], [26], [30], [32], and [33] for the valuation of American-type and barrier options. This randomisation method is based on the fact that, according to the law of large numbers, the average of independent exponential random variables with mean $t$ converges to $t$. An average of $n$ such exponential random variables is equal in distribution to a $\operatorname{gamma}(n, n / t)$ random variable $\Gamma_{n, n / t}$, which has mean $t$ and variance $t^{2} / n$. As observed in [17, Chapter VII.6], the approximation of the value $f(t)$ of a continuous bounded function $f$ at $t>0$ by the expectation $\mathbb{E}\left[f\left(\Gamma_{n, n / t}\right)\right]$ of $f$ evaluated at the random time $\Gamma_{n, n / t}$ is asymptotically exact. Since $\Gamma_{n, n / t}$ converges to a point mass at $t$, it follows that the expectation $\mathbb{E}\left[f\left(\Gamma_{n, n / t}\right)\right]$ converges to $f(t)$ as $n$ tends to $\infty$. As regards the rate of convergence, the form of the probability density function (PDF) of $\Gamma_{n, n / t}$ implies that, in the case that $f$ is $C^{2}$ at $t$, the decay of the error $\mathbb{E}\left[f\left(\Gamma_{n, n / t}\right)\right]-f(t)$ is linear in $1 / n$, in line with $[3$, Theorem 6$]$, and that, moreover, $\mathbb{E}\left[f\left(\Gamma_{n, n / t}\right)\right]$ admits the following expansion if the function $f$ is $C^{2 k}$ at $t$ :

$$
\mathbb{E}\left[f\left(\Gamma_{n, n / t}\right)\right]-f(t)=\sum_{m=1}^{k} b_{m}(t)\left(\frac{1}{n}\right)^{m}+o\left(n^{-k}\right) \quad \text { as } n \rightarrow \infty,
$$

for certain functions $b_{1}, \ldots, b_{k}$ (given in Theorem 3.1 below). We apply this expansion to functions $f(t)$ that are equal to the expectations of path-dependent functionals of Lévy bridges living on the time-interval $[0, t]$. We note that $\mathbb{E}\left[f\left(\Gamma_{n, n / t}\right)\right]$ is equal to the expectation of the corresponding path-functional of the Lévy process $X$ pinned down at an independent random time that is equal in distribution to $\Gamma_{n, n / t}$. For the path-dependent functionals that we consider (namely, first-passage times and occupation times of sets) the corresponding functions $f$ are sufficiently smooth, so that the use of the Richardson extrapolation is fully justified. Furthermore, it holds (see Theorem A.1) that the density functions $D_{n}(x, y)$ and $\Omega_{n}(x, y), n \in \mathbb{N}$, given by $D_{n, q}(x, y) \mathrm{d} y=\mathbb{P}\left(\bar{X}_{\Gamma_{n, q}} \leq x, X_{\Gamma_{n, q}} \in \mathrm{d} y\right)$ and $\Omega_{n, q}(x, y) \mathrm{d} x \mathrm{~d} y=$ $\mathbb{E}\left[\int_{0}^{\Gamma_{n, q}} \mathbf{1}_{\left\{X_{u} \in \mathrm{d} x, X_{\Gamma_{n, q}} \in \mathrm{d} y\right\}} \mathrm{d} u\right]$ corresponding to a random horizon $\Gamma_{n, n / t}$ satisfy the following recursions for $x, y \in \mathbb{R}$ and $n \in \mathbb{N}$ :

$$
\begin{gathered}
D_{n+1, q}(x, y)=\int_{-\infty}^{x} D_{n, q}(x-w, y-w) D_{1, q}(x, w) \mathrm{d} w, \quad \max \{y, 0\} \leq x \\
\Omega_{n+1, q}(x, y)=\int_{-\infty}^{\infty}\left[\Omega_{1, q}(x, w) u_{n, q}(y-w)+\Omega_{n, q}(x-w, y-w) u_{1, q}(w)\right] \mathrm{d} w
\end{gathered}
$$


where $u_{n, q}$ is the PDF of the random variable $X_{\Gamma_{n, q}}$. For the dense class of mixed-exponential Lévy processes (see Definition 2.1 below) we present explicit solutions to these recursions. By way of numerical illustration the method was implemented for a number of models in this class, and the numerical outcomes are reported in Section 4, confirming the theoretically predicted rates of decay of the error. We observed that the Richardson extrapolation based on a small number (about ten) recursive steps already yields highly accurate approximations.

Markov bridge method. We combine subsequently these approximations with a continuoustime EM scheme to estimate the conditional expectations $\widetilde{F}$ corresponding to the first-passage times and occupation times of sets of a stochastic volatility process with jumps. To illustrate the effectiveness of the method, we evaluated a barrier option and a range note under a Batestype model using the proposed Markov bridge Monte Carlo scheme, and report the results in Section 5. The rates of decay of the error that we find numerically in the case of barrier options are in line with the corresponding error estimates that were established in [21] for the case of killed diffusion processes.

The remainder of this paper is organized as follows. In Section 2 explicit expressions are derived for the first-passage probabilities and expected occupation times of a mixed-exponential Lévy process. Section 3 is devoted to error estimates and numerical illustrations are presented in Section 4. Section 5 contains a Markov bridge sampling method based on the randomisation method and numerical illustrations. The proof of the recursions (1.2) and (1.3) is deferred to Appendix A.

\section{Maximum and occupation time of mixed-exponential Lévy models}

In this section we show that the recursions in (1.2) and (1.3) admit explicit solutions in the case that the Lévy process $X$ is a mixed-exponential jump-diffusion, the definition of which we recall next.

Definition 2.1. (i) A random variable has a mixed-exponential density if it has $\operatorname{PDF} f$ given by

$$
f(x)=\sum_{i=1}^{m^{+}} p_{i}^{+} \alpha_{i}^{+} \mathrm{e}^{-\alpha_{i}^{+} x} \mathbf{1}_{(0, \infty)}(x)+\sum_{j=1}^{m^{-}} p_{j}^{-} \alpha_{j}^{-} \mathrm{e}^{-\alpha_{j}^{-}|x|} \mathbf{1}_{(-\infty, 0)}(x),
$$

where $\sum_{k=1}^{m^{ \pm}} p_{k}^{ \pm}=q^{ \pm}, q^{+}+q^{-}=1$, and $-\alpha_{m^{-}}^{-}<\cdots<-\alpha_{1}^{-}<0<\alpha_{1}^{+}<\cdots<\alpha_{m^{+}}^{+}$.

(ii) A Lévy process $X=\left\{X_{t}, t \in \mathbb{R}_{+}\right\}$is a mixed-exponential jump-diffusion (MEJD) if it is of the form

$$
X_{t}=\mu t+\sigma W_{t}+\sum_{i=1}^{N_{t}} U_{i}
$$

where $\mu$ is a real number and $\sigma$ is strictly positive, $W$ is a standard Brownian motion, $N$ is a Poisson process with intensity $\lambda$, and the jump sizes $\left\{U_{i}, i \in \mathbb{N}\right\}$ are independent and identically distributed (i.i.d.) with mixed-exponential density. Here, the collections $W=\left\{W_{t}, t \in \mathbb{R}_{+}\right\}$, $N=\left\{N_{t}, t \in \mathbb{R}_{+}\right\}$, and $\left\{U_{i}, i \in \mathbb{N}\right\}$ are independent.

Remark 2.1. (i) Including in Definition 2.1 the additional restriction that the weights $p_{k}^{ \pm}$ are nonnegative, the Lévy process is a hyper-exponential jump-diffusion (HEJD). While HEJD processes are dense in the class of all Lévy processes with a completely monotone Lévy density, the collection of MEJDs is dense in the class of all Lévy processes, in the sense of weak convergence of probability measures; see [9]. 
(ii) The parameters $\left\{p_{k}^{ \pm}, k=1, \ldots, m^{ \pm}\right\}$cannot be chosen arbitrarily but need to satisfy a restriction to guarantee that $f$ is a PDF. Necessary and sufficient conditions for $f$ to be a PDF are

$$
p_{1}^{ \pm}>0, \quad \sum_{k=1}^{m^{ \pm}} p_{k}^{ \pm} \alpha_{k}^{ \pm} \geq 0, \quad \text { and } \quad \sum_{k=1}^{l} p_{k}^{ \pm} \alpha_{k}^{ \pm} \geq 0 \quad \text { for all } l=1, \ldots, m^{ \pm},
$$

respectively. For a proof of these results and alternative conditions, see [5]. In Section 5 we will impose the additional condition $\alpha_{1}^{+}>1$, which ensures that the expectation $\mathbb{E}\left[S_{t}\right]$ of the exponential Lévy process $S_{t}=\exp \left\{X_{t}\right\}$ is finite for any nonnegative $t$.

(iii) Samples can be drawn from the mixed-exponential distribution by using the acceptancerejection method (see [37]) and taking as the instrumental distribution a double-exponential distribution. The double-exponential density multiplied by a constant will dominate the original mixed-exponential density. In the next section this method was used to obtain the Monte Carlo results.

(iv) Since $\sigma$ is strictly positive, Assumption A.1 given in Appendix A is satisfied for the MEJD process $X$, and $X_{\Gamma_{n, q}}, n \in \mathbb{N}, q>0$, has a density by Lemma A.2.

From the definition of the MEJD process $X$ it is straightforward to verify that the characteristic exponent $\Psi(s)=-\log \mathbb{E}\left[\mathrm{e}^{\mathrm{i} s X_{1}}\right]$ is a rational function of the form

$$
\Psi(s)=-\mathrm{i} \mu s+\frac{\sigma^{2} s^{2}}{2}-\lambda\left(\sum_{i=1}^{m^{+}} p_{i}^{+} \frac{\alpha_{i}^{+}}{\alpha_{i}^{+}-\mathrm{i} s}+\sum_{j=1}^{m^{-}} p_{j}^{-} \frac{\alpha_{j}^{-}}{\alpha_{j}^{-}+\mathrm{i} s}-1\right), \quad s \in \mathbb{R} .
$$

The distributions of $X$, the running supremum $\bar{X}$, and the running infimum $\underline{X}$ at the random time $\Gamma_{1, q}$ and also the functions $D_{1, q}$ and $\Omega_{1, q}$ can be expressed, as we shall see below, in terms of the roots $\left\{\rho_{k}^{+}, k=1, \ldots, m^{+}+1\right\}$ and $\left\{\rho_{k}^{-}, k=1, \ldots, m^{-}+1\right\}$ with positive and negative real parts of the Cramér-Lundberg equation

$$
q+\Psi(-\mathrm{i} s)=0, \quad q>0 .
$$

For the MEJD $X$ the Wiener-Hopf factors $\Psi_{q}^{+}$and $\Psi_{q}^{-}$can be identified explicitly. It is well known that $\Psi_{q}^{+}(\theta)$ and $\Psi_{q}^{-}(\theta)$ have neither 0 s nor poles on the half-planes $\{\operatorname{Im}(z)>0\}$ and $\{\operatorname{Im}(z)<0\}$, respectively, as a consequence of the fact that $\Psi_{q}^{+}$and $\Psi_{q}^{-}$are the characteristic functions of infinitely divisible distributions supported on the positive and negative half-lines respectively; see [39, Chapter 9]. In particular, using the fact that $\Psi_{q}^{+}(\theta)$ and $\Psi_{q}^{-}(\theta)$ satisfy $q /(q+\Psi(\theta))=\Psi_{q}^{+}(\theta) \Psi_{q}^{-}(\theta)$ for $\theta \in \mathbb{R}$, the Wiener-Hopf factors of an MEJD can be identified as certain rational functions; see [34].

Lemma 2.1. Let $q>0$ be given. The functions $\Psi_{q}^{+}$and $\Psi_{q}^{-}$are given explicitly by

$$
\begin{aligned}
& \Psi_{q}^{+}(s):=\prod_{i=1}^{m^{+}}\left(1-\frac{\mathrm{i} s}{\alpha_{i}^{+}}\right) \prod_{i=1}^{m^{+}+1}\left(1-\frac{\mathrm{i} s}{\rho_{i}^{+}(q)}\right)^{-1}, \\
& \Psi_{q}^{-}(s):=\prod_{j=1}^{m^{-}}\left(1+\frac{\mathrm{i} s}{\alpha_{j}^{-}}\right) \prod_{j=1}^{m^{-}+1}\left(1-\frac{\mathrm{i} s}{\rho_{j}^{-}(q)}\right)^{-1} .
\end{aligned}
$$


The fact that the Wiener-Hopf factors $\Psi_{q}^{+}$and $\Psi_{q}^{-}$are rational functions implies that, when the roots of the Cramér-Lundberg equation are distinct, the running supremum $\bar{X}_{\Gamma_{1, q}}$ and infimum $\underline{X}_{\Gamma_{1, q}}$ of $X$ at $\Gamma_{1, q}$, where $\bar{X}_{t}:=\sup _{s \leq t} X_{s}$ and $\underline{X}_{t}:=\inf _{s \leq t} X_{s}$ denote the running supremum and infimum of $X$ at $t \in \mathbb{R}_{+}$, also follow mixed-exponential distributions.

Lemma 2.2. Let $q>0$ be given and suppose that the roots of (2.1) are distinct. The random variables $\bar{X}_{\Gamma_{1, q}},-\underline{X}_{\Gamma_{1, q}}$, and $X_{\Gamma_{1, q}}$ have mixed-exponential distributions with densities $\bar{u}_{1, q}$, $\underline{u}_{1, q}$, and $u_{1, q}$ given by

$$
\begin{gathered}
\bar{u}_{1, q}(z)=\sum_{i=1}^{m^{+}+1} A_{i}^{+}(q) \rho_{i}^{+}(q) \mathrm{e}^{-\rho_{i}^{+}(q) z}, \quad \underline{u}_{1, q}(z)=\sum_{j=1}^{m^{-}+1} A_{j}^{-}(q)\left(-\rho_{j}^{-}(q)\right) \mathrm{e}^{\rho_{j}^{-}(q) z}, \\
u_{1, q}(x)=\sum_{i=1}^{m^{+}+1} B_{i}(q) \mathrm{e}^{-\rho_{i}^{+}(q) x} \mathbf{1}_{(0, \infty)}(x)+\sum_{j=1}^{m^{-}+1} C_{j}(q) \mathrm{e}^{-\rho_{j}^{-}(q) x} \mathbf{1}_{(-\infty, 0)}(x)
\end{gathered}
$$

for $z \in \mathbb{R}_{+}$and $x \in \mathbb{R}$ with, for $i=1, \ldots, m^{+}+1$ and $j=1, \ldots, m^{-}+1$,

$$
\begin{array}{ll}
A_{i}^{+}(q):=\frac{\prod_{k=1}^{m^{+}}\left(1-\rho_{i}^{+}(q) / \alpha_{k}^{+}\right)}{\prod_{k \neq i}\left(1-\rho_{i}^{+}(q) / \rho_{k}^{+}(q)\right)}, \quad A_{j}^{-}(q):=\frac{\prod_{k=1}^{m^{-}}\left(1+\rho_{j}^{-}(q) / \alpha_{k}^{-}\right)}{\prod_{k \neq j}\left(1-\rho_{j}^{-}(q) / \rho_{k}^{-}(q)\right)}, \\
B_{i}(q):=A_{i}^{+}(q) \Psi_{q}^{-}\left(\rho_{i}^{+}(q)\right) \rho_{i}^{+}(q), & C_{j}(q):=A_{j}^{-}(q) \Psi_{q}^{+}\left(\rho_{j}^{-}(q)\right)\left(-\rho_{j}^{-}(q)\right),
\end{array}
$$

where we define $A_{k}^{ \pm} \equiv 1$ in the $m^{ \pm}=0$ case (i.e. if there are no positive/negative jumps).

Proof. It is straightforward to verify that the coefficients of the function $\left(1-\text { is } / \rho_{i}^{+}(q)\right)^{-1}$ in the partial-fraction decompositions of the functions $q /(q+\Psi(s))$ and $\Psi_{q}^{+}(s)$ are given by $C_{i}(q)$ and $A_{i}^{+}(q)$, respectively, while the coefficients of the function $\left(1-\mathrm{i} s / \rho_{j}^{-}(q)\right)^{-1}$ in the partial-fraction decompositions of the functions $q /(q+\Psi(s))$ and $\Psi_{q}^{-}(s)$ are given by $B_{j}(q)$ and $A_{j}^{-}(q)$, respectively. Subsequently inverting the Fourier transforms $\left(1-\mathrm{is} / \rho_{i}^{+}(q)\right)^{-1}$ and $\left(1-\mathrm{i} s / \rho_{j}^{-}(q)\right)^{-1}$ yields the stated expressions for the densities of $\bar{X}_{\Gamma_{1, q}},-\underline{X}_{\Gamma_{1, q}}$, and $X_{\Gamma_{1, q}}$.

The functions $\Omega_{n, q}$ and $D_{n, q}$ and the density $u_{n, q}$ can be explicitly identified by combining the forms of the functions $\Omega_{1, q}$ and $D_{1, q}$ (identified below) with the recursive relations in (1.2) and (1.3). From the form of these recursive relations it follows that the functions $\Omega_{n, q}, D_{n, q}$, and $u_{n, q}$ can be expressed as linear combinations of exponentials with the weights given by certain polynomials-the explicit expressions are given in the following result.

Consider the polynomials $\widetilde{P}_{k, i, n}^{ \pm}, \widetilde{P}_{i, j, k, n}^{ \pm}$, and real numbers $\widetilde{c}_{i, j, n}^{ \pm}$defined by

$$
\begin{gathered}
\int_{0}^{x} P_{k, n}^{+}(y) \mathrm{e}^{-\rho_{k}^{+} y-\rho_{i}^{+}(x-y)} \mathrm{d} y=\mathrm{e}^{-\rho_{k}^{+} x} \widetilde{P}_{k, i, n}^{+}(x)-\mathrm{e}^{-\rho_{i}^{+} x} \widetilde{c}_{k, i, n}^{+}, \\
\int_{x}^{0} P_{k, n}^{-}(y) \mathrm{e}^{-\rho_{k}^{-} y-\rho_{i}^{-}(x-y)} \mathrm{d} y=\mathrm{e}^{-\rho_{k}^{-} x} \widetilde{P}_{k, i, n}^{-}(x)-\mathrm{e}^{-\rho_{i}^{-} x} \widetilde{c}_{k, i, n}^{-}, \\
\int_{0}^{x} \mathrm{e}^{\rho_{i}^{+}(z-x)} P_{i, j, n}(x-z, y-z) u_{1}(z) \mathrm{d} z=\sum_{k=1}^{m^{+}+1} \widetilde{P}_{i, j, k, n}^{+}(x, y) \mathrm{e}^{-\rho_{k}^{+} x}, \\
\int_{0}^{x} \mathrm{e}^{-\rho_{j}^{-}(x-z)} u_{n}(z) \mathrm{d} z=\sum_{k=1}^{m^{-}+1} \widetilde{P}_{i, j, k, n}^{-}(x) \mathrm{e}^{-\rho_{k}^{-} x},
\end{gathered}
$$


where we denoted $\rho_{h}^{+}=\rho_{h}^{+}(q)$ and $\rho_{h}^{-}=\rho_{h}^{-}(q)$, and $P_{k, n}^{+}$and $P_{k, n}^{-}$are the polynomials to be defined shortly. The fact that there exist polynomials and constants satisfying the above relations follows by repeated integration by parts. By induction the following expressions for the functions $u_{n, q}, D_{n, q}$, and $\Omega_{n, q}$ can be derived.

Proposition 2.1. For any $n \in \mathbb{N} \cup\{0\}$, we have

$$
\begin{gathered}
u_{n+1, q}(x)=\sum_{k=1}^{m^{+}+1} P_{k, n+1}^{+}(x) \mathrm{e}^{-\rho_{k}^{+} x} \mathbf{1}_{(0, \infty)}(x)+\sum_{k=1}^{m^{-}+1} P_{k, n+1}^{-}(x) \mathrm{e}^{-\rho_{k}^{-} x} \mathbf{1}_{(-\infty, 0)}(x), \\
D_{n+1, q}(z, y)=u_{n+1, q}(y)-\sum_{i=1}^{m^{+}+1} \sum_{j=1}^{m^{-}+1} P_{i, j, n+1}(z, y) \mathrm{e}^{-\rho_{j}^{-}(y-z)-\rho_{i}^{+} z}, \quad z \geq y, \\
\Omega_{n+1, q}(x, y)=q^{-(n+1)} \sum_{k=1}^{n+1} u_{n+2-k, q}(x) u_{k, q}(y-x)
\end{gathered}
$$

for $x, y \in \mathbb{R}$, and $z \in \mathbb{R}_{+}$with, as before, $\rho_{j}^{-}=\rho_{j}^{-}(q)$ and $\rho_{i}^{+}=\rho_{i}^{+}(q)$, and with $P_{k, 1}^{+} \equiv$ $B_{k}(q), P_{k, 1}^{-} \equiv C_{k}(q)$ and $P_{i, j, 1} \equiv E_{i j}(q) /\left(\rho_{j}^{-}-\rho_{i}^{+}\right):=A_{i}^{+}(q) A_{j}^{-}(q) \rho_{i}^{+}(q) \rho_{j}^{-}(q) /\left(\rho_{j}^{-}-\right.$ $\left.\rho_{i}^{+}\right)$, and where $P_{k, n+1}^{ \pm}$and $P_{i, j, n+1}$ are polynomials and $c_{k, i, n}^{ \pm}$are real numbers that are defined recursively for $n \in \mathbb{N}$ as

$$
\begin{aligned}
& P_{k, n+1}^{+}(x)=\sum_{r=1}^{m^{-}+1}\left(C_{r}(q) \int_{0}^{\infty} \mathrm{e}^{\left(\rho_{r}^{-}-\rho_{k}^{+}\right) z} P_{k, n}^{+}(x+z) \mathrm{d} z+B_{k}(q) c_{k, r, n}^{-}\right) \\
& +\sum_{r=1}^{m^{+}+1} B_{r}(q)\left(\widetilde{P}_{k, r, n}^{+}(x)-\widetilde{c}_{r, k, n}^{+}\right) \\
& P_{k, n+1}^{-}(x)=\sum_{r=1}^{m^{+}+1}\left(B_{r}(q) \int_{-\infty}^{0} \mathrm{e}^{\left(\rho_{r}^{+}-\rho_{k}^{-}\right) z} P_{k, n}^{-}(x+z) \mathrm{d} z+C_{k}(q) c_{k, r, n}^{+}\right) \\
& +\sum_{r=1}^{m^{-}+1} C_{r}(q)\left(\widetilde{P}_{k, r, n}^{-}(x)-\widetilde{c}_{r, k, n}^{-}\right) \\
& P_{i, j, n+1}(x, y)=\int_{-\infty}^{0} P_{i, j, n}(x-z, y-z) \mathrm{e}^{\rho_{i}^{+} z} u_{1}(z) \mathrm{d} z+\sum_{k=1}^{m^{+}+1} \widetilde{P}_{k, j, i, n}^{+}(x, y) \\
& -\sum_{k=1}^{m^{-}+1} \widetilde{P}_{i, k, j, n}^{-}(y-x)+B_{i}(q) \int_{0}^{\infty} P_{j, n}^{-}(y-x-z) \mathrm{e}^{\left(\rho_{j}^{-}-\rho_{i}^{+}\right) z} \mathrm{~d} z \\
& +\frac{E_{i, j}(q)}{\rho_{j}^{-}-\rho_{i}^{+}} \int_{0}^{\infty} u_{n, q}(z) \mathrm{e}^{\rho_{j}^{-} z} \mathrm{~d} z \\
& -\sum_{k=1}^{m^{+}+1} \sum_{l=1}^{m^{-}+1} \frac{E_{k, l}(q)}{\rho_{l}^{-}-\rho_{k}^{+}} \int_{-\infty}^{0} P_{i, j, n}(-z, y-x-z) \mathrm{e}^{\rho_{i}^{+} z-\rho_{l}^{-} z} \mathrm{~d} z, \\
& c_{k, r, n}^{-}=\int_{-\infty}^{0} \mathrm{e}^{\left(\rho_{k}^{+}-\rho_{r}^{-}\right) z} P_{r, n}^{-}(z) \mathrm{d} z, \quad c_{k, r, n}^{+}=\int_{0}^{\infty} \mathrm{e}^{\left(\rho_{k}^{-}-\rho_{r}^{+}\right) z} P_{r, n}^{+}(z) \mathrm{d} z .
\end{aligned}
$$


Proof. By combining the identity

$$
\mathbb{P}\left[\bar{X}_{\Gamma_{1, q}} \in \mathrm{d} x, x-X_{\Gamma_{1, q}} \in \mathrm{d} z\right]=\mathbb{P}\left[\bar{X}_{\Gamma_{1, q}} \in \mathrm{d} x\right] \mathbb{P}\left[-\underline{X}_{\Gamma_{1, q}} \in \mathrm{d} z\right], \quad x, z \in \mathbb{R}_{+}
$$

(which follows from the Wiener-Hopf factorisation of $X$ ) with Lemma 2.2 and performing a one-dimensional integration, we obtain the expression for the function $D_{1, q}$. The Markov property and stationarity of increments yields $\Omega_{1, q}(x, y)=q^{-1} u_{1, q}(y-x) u_{1, q}(x)$, whence we have the form of the function $\Omega_{1, q}$ by inserting the expression for $u^{q}$ in Lemma 2.2. The expressions for $u_{n+1, q}, D_{n+1, q}$, and $\Omega_{n+1, q}$ follow by induction with respect to $n$, utilising

(i) the fact that $u_{n+1, q}$ is equal to the convolution of $u_{n, q}$ and $u_{1, q}$, as a consequence of the independence and stationarity of the increments of $X$,

(ii) the form of $D_{1, q}$ and the recursive relation in (1.2), and

(iii) the form of $\Omega_{1, q}$ and the recursive relation in (1.3).

\section{Convergence and error estimates}

The randomisation method consists of approximating the value $f(t)$ of a function $f$ at time $t>0$ by the expectation $\mathbb{E}\left[f\left(\Gamma_{n, n / t}\right)\right]$ of $f$ evaluated at a random time $\Gamma_{n, n / t}$ that follows a gamma distribution with expectation $\mathbb{E}\left[\Gamma_{n, n / t}\right]=t$ and variance $\mathbb{E}\left[\left(\Gamma_{n, n / t}-t\right)^{2}\right]=t^{2} / n$. Since the random variables $\Gamma_{n, n / t}$ converge in distribution to $t$ as $n$ tends to $\infty$, the error $\mathbb{E}\left[f\left(\Gamma_{n, n / t}\right)\right]-f(t)$ converges to 0 for any bounded and continuous function $f$. The error can be expanded in terms of powers of $1 / n$ provided that $f$ is sufficiently smooth, as shown in the following result.

Theorem 3.1. Let $k$ be a given nonnegative integer and consider $f \in C^{2 k+2}\left(\mathbb{R}_{+}\right)$. There exist functions $b_{1}, \ldots, b_{k+1}: \mathbb{R}_{+} \rightarrow \mathbb{R}$ such that for any $t \in \mathbb{R}_{+}$, we have

$$
n^{k+1}\left[\mathbb{E}\left[f\left(\Gamma_{n, n / t}\right)\right]-f(t)-\sum_{m=1}^{k} b_{m}(t)\left(\frac{1}{n}\right)^{m}\right]=b_{k+1}(t)+o(1) \text { as } n \rightarrow \infty \text {. }
$$

In particular, denoting by $f^{(m)}$ the mth derivative of $f$, we have

$$
\begin{gathered}
b_{1}(t)=\frac{t^{2}}{2} f^{(2)}(t), \quad b_{2}(t)=\frac{t^{4}}{8} f^{(4)}(t)+\frac{t^{3}}{3} f^{(3)}(t), \\
b_{3}(t)=\frac{t^{6}}{48} f^{(6)}(t)+\frac{t^{5}}{6} f^{(5)}(t)+\frac{t^{4}}{4} f^{(4)}(t) .
\end{gathered}
$$

Remark 3.1. (i) Theorem 3.1 implies that for $f \in C^{2}\left(\mathbb{R}_{+}\right)$the error of the approximation of $f(t)$ by $\mathbb{E}\left[f\left(\Gamma_{n, n / t}\right)\right]$ decays linearly, that is, $\mathbb{E}\left[f\left(\Gamma_{n, n / t}\right)\right]-f(t)=b_{1}(t) / n+o(1 / n)$ as $n$ tends to $\infty$.

(ii) Theorem 3.1 also provides a justification for the use of the Richardson extrapolation to increase the speed of convergence if the function $f$ is sufficiently smooth. Since the error of the approximation is given in terms of positive integer powers of $1 / n$, the Richardson extrapolation that utilises the first $N$ values $\mathbb{E}\left[f\left(\Gamma_{1,1 / t}\right)\right], \ldots, \mathbb{E}\left[f\left(\Gamma_{N, N / t}\right)\right]$ is explicitly given by

$$
P_{1: N}=\sum_{k=1}^{N} \frac{(-1)^{N-k} k^{N}}{k !(N-k) !} \mathbb{E}\left[f\left(\Gamma_{k, k / t}\right)\right]
$$


see [35, Section 1.3] for a derivation of this formula. In particular, note that in order to deploy the extrapolation (3.2) it suffices to know the existence of functions $b_{m}$ such that (3.1) holds and it is not required to find their explicit form. In the $f \in C^{2 k+2}\left(\mathbb{R}_{+}\right), k<N$ case Theorem 3.1 implies that the error $P_{1: N}-f(t)$ of the interpolation $P_{1: N}$ is $o\left(N^{-k-1}\right)$. In particular, if $f$ is $C^{\infty}$ then the error $P_{1: N}-f(t)$ is $O\left(N^{-k-1}\right)$ for every $k$, as $N$ tends to $\infty$. See [40] for background on the theory of extrapolation and interpolation.

Proof of Theorem 3.1. To the best of the authors' knowledge a proof of this result does not exist in the literature; here we provide a brief proof. Taylor's theorem and the fact that $f \in C^{2 k+2}$ imply

$$
f(s)-f(t)=\sum_{m=1}^{2 k+1} \frac{(s-t)^{m}}{m !} f^{(m)}(t)+R(s, t),
$$

where the remainder term is given by $R(s, t)=\left((s-t)^{2 k+2} /(2 k+2) !\right) f^{(2 k+2)}(\xi)$ for some $\xi$ between $s$ and $t$. Replacing $s$ by the independent gamma random variable $\Gamma_{n, n / t}$, we obtain

$$
\mathbb{E}\left[f\left(\Gamma_{n, n / t}\right)-f(t)\right]=\sum_{m=2}^{2 k+1} \frac{a_{m, n}}{m !} f^{(m)}(t)+\mathbb{E}\left[R\left(\Gamma_{n, n / t}, t\right)\right]
$$

with $a_{m, n}=\mathbb{E}\left[\left(\Gamma_{n, n / t}-t\right)^{m}\right]$, where we have $a_{1, n}=0$ as the expectation $\mathbb{E}\left[\Gamma_{n, n / t}\right]$ is equal to $t$. The numbers $a_{m, n}$ are equal to $a_{m, n}=\left.\left(\mathrm{d}^{m} / \mathrm{d} u^{m}\right)\right|_{u=0} M(u)$, where $M$ denotes the momentgenerating function of the random variable $\Gamma_{n, n / t}-t$ which is given by

$$
M(u)=\left(1-\frac{u t}{n}\right)^{-n} \exp \{-u t\}, \quad u<\frac{n}{t} .
$$

In particular, it follows from the form of $M$ that the $a_{m, n}$ are linear combinations of positive integer powers of $1 / n$. Reordering of terms and straightforward manipulations result in the identity in (3.1).

We next turn to the problem of approximation of the distribution of the supremum and the expected occupation time of the set $(-\infty, x]$ of the Lévy bridge process $X^{(0,0) \rightarrow(t, y)}$ from $(0,0)$ to $(t, y)$ (its definition is given in Appendix A):

$$
\vec{d}_{t}(x, y):=\mathbb{P}\left(\bar{X}^{(0,0) \rightarrow(t, y)} \leq x\right), \quad \vec{\omega}_{t}(x, y):=\mathbb{E}\left[\int_{0}^{t} 1_{\left\{X_{u}^{(0,0) \rightarrow(t, y)} \leq x\right\}} \mathrm{d} u\right],
$$

with $\bar{X}^{(0,0) \rightarrow(t, y)}:=\sup _{u \in[0, t]} X_{u}^{(0,0) \rightarrow(t, y)}$. By the spatial and temporal homogeneity of $X$, the corresponding quantities in the case of a general starting point $(s, z)$ are given in terms of $\vec{d}$ and $\vec{\omega}$ by $\vec{d}_{t-s}(x-z, y-z)$ and $\vec{\omega}_{t-s}(x-z, y-z)$. The approximations of $\vec{d}$ and $\vec{\omega}$ are given in terms of the randomised bridge process $X^{(0,0) \rightarrow\left(\Gamma_{n, q}, y\right)}$ (see Appendix A) as

$$
\vec{D}_{q}^{(n)}(x, y):=\mathbb{P}\left(\bar{X}^{(0,0) \rightarrow\left(\Gamma_{n, q}, y\right)} \leq x\right), \quad \vec{\Omega}_{q}^{(n)}(x, y):=\mathbb{E}\left[\int_{0}^{\Gamma_{n, q}} 1_{\left\{X_{u}^{(0,0) \rightarrow\left(\Gamma_{n, q}, y\right)} \leq x\right\}} \mathrm{d} u\right] .
$$

Error estimates for these randomised bridge approximations are given as follows.

Corollary 3.1. Let $x, y \in \mathbb{R}$ and $t>0$. For some constants $C^{d}$ and $C^{\omega}$, we have, for all positive integers $n$,

$$
\left|\vec{D}_{n / t}^{(n)}(x, y)-\vec{d}_{t}(x, y)\right| \leq \frac{C^{d}}{n}, \quad\left|\vec{\Omega}_{n / t}^{(n)}(x, y)-\vec{\omega}_{t}(x, y)\right| \leq \frac{C^{\omega}}{n} .
$$


Proof. Since the distribution of $X_{t}$ has a continuous density $p_{t}(y)$ and $s \mapsto \vec{d}_{s}(x, y)$, $s \mapsto \vec{\omega}_{s}(x, y)$, and $s \mapsto p_{s}(y)$ are $C^{2}$ at $s=t$ with $p_{t}(y)>0$, the estimates in (3.3) follow by applying Theorem 3.1 to the functions $t \mapsto \vec{d}_{t}(x, y) p_{t}(y), t \mapsto \vec{\omega}_{t}(x, y) p_{t}(y)$, and $t \mapsto p_{t}(y)$.

\section{Illustration: first-passage time probabilities and occupation times}

To provide a numerical illustration of the randomisation method, we implemented the recursive formulae (given in Proposition 2.1) to approximate the following expectations of path-dependent functionals:

$$
\mathbb{P}\left(\sup _{u \in[0, t]} X_{u}^{(0, x) \rightarrow(t, y)} \leq z\right), \quad \mathbb{E}\left[\int_{0}^{t} 1_{\left\{X_{u}^{(0, x) \rightarrow(t, y)} \in(a, b)\right\}} \mathrm{d} u\right],
$$

where $z=1.2, a=1.05, b=1.25$, with $t=1, x=1, y=1.1$, for the case that the underlying Lévy process $X$ is equal to a HEJD process with typical parameters, which are detailed in Table 1; see [41, Chapter 3] for additional numerical examples. The outcomes are reported in Table 2 and Figure 1. In Table 2 the values are listed of the first-passage time probabilities and the expected occupation times of the randomised Lévy bridges corresponding to a $\Gamma(n, n)$-randomisation of the fixed time $T=1$ for a number of values of $n$. We also report the results obtained by applying a Richardson extrapolation $P_{1: n}$ of order $n$, using the first $n$ outcomes (defined in (3.2)). The logarithms of the corresponding absolute errors are plotted in Figure 1. The errors were computed with respect to the value $P_{1: 11}$ that was obtained after Richardson's extrapolation with $n=11$ stages.

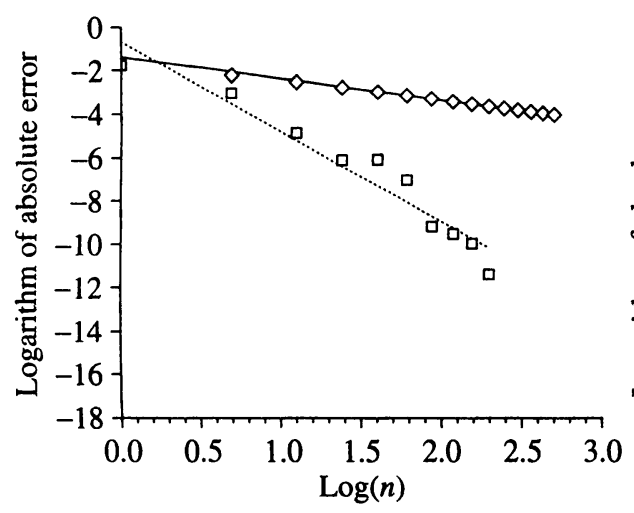

(a)

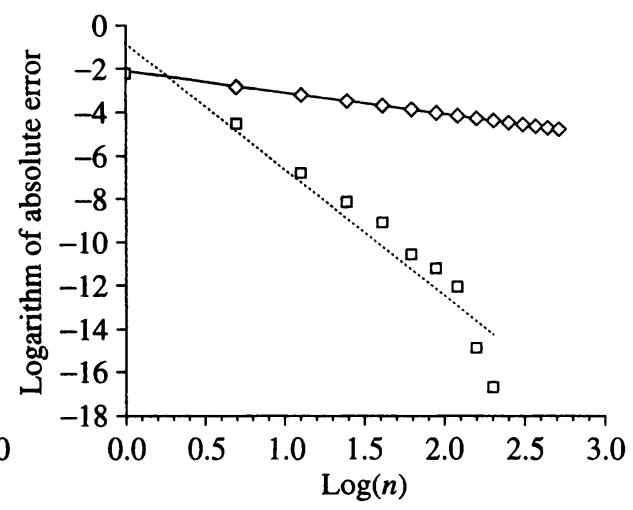

(b)

$\square$ Recursive $\diamond$ Extrapolated - OLS Recursive …… OLS Extrapolated

FIGURE 1: The logarithms of the absolute errors of the outcomes generated by the recursive algorithm for (a) the one-sided first-passage probabilities and (b) the expected occupation time under the HEJD processes as a function of $n$, where $n$ is the number of steps in the recursions. In each figure the errors of the recursive values and the Richardson extrapolated values are displayed. Also OLS estimations of either series of results are plotted (in the case of the unextrapolated values the OLS line was estimated using the last six values only). The slopes of the solid lines in (a) and (b) are given by -0.98 and -0.99 , respectively. The starting point of the bridge is 1.0 , the end point is 1.1 , the barrier level is 1.2 , and the range is $(1.05,1.25)$. In all cases the Lévy bridge process is assumed to start at time 0 and to end at time 1 .

The model parameters that were used are given in Table 1. 
TABLE 1: The model parameters used throughout the paper. The parameters for the Kou model are taken from [29], the parameters for the HEJD model from [24], and the parameters for the MEJD model from [12] (which for the latter two models have been reexpressed using our notation).

\begin{tabular}{lrc}
\hline & KOU & HEJD \\
\hline$\sigma$ & 0.2 & $\sqrt{0.042}$ \\
$\lambda$ & 3.0 & 11.5 \\
$\alpha^{+}$ & 50.0 & $(5,10,15,25,30,60,80)$ \\
$\alpha^{-}$ & 25.0 & $(5,10,15,25,30,60,80)$ \\
$p^{+}$ & 0.3 & $(0.05,0.05,0.1,0.6,1.2,1.9,6.1) * 0.51 / \lambda$ \\
$p^{-}$ & 0.7 & $(0.5,0.3,1.1,0.8,1,4,2.3) * 0.64 / \lambda$ \\
\hline & & MEJD \\
\hline$\sigma$ & 0.2 & 0.2 \\
$\lambda$ & 3.0 & 1.0 \\
$\alpha^{+}$ & 50.0 & $(213.0215,236.0406,237.1139,939.7441,939.8021)$ \\
$\alpha^{-}$ & 25.0 & $(213.0215,236.0406,237.1139,939.7441,939.8021)$ \\
$p^{+}$ & 0.3 & $(4.36515,1.0833,-5,0.0311,0.02045)$ \\
$p^{-}$ & 0.7 & $(4.36515,1.0833,-5,0.0311,0.02045)$ \\
\hline
\end{tabular}

TABLE 2: Approximations of one-sided first-passage time (FPT) probabilities and expected occupation times obtained recursively $\left(P_{n}\right)$ and with Richardson extrapolation $\left(P_{1: n}\right)$ for the HEJD model as a function of $n$, where $n$ is the number of recursions. The starting point of the bridge is assumed to be 1.0, the end point is 1.1 , the barrier level is 1.2 , and the range is $(1.05,1.25)$. In all cases the Lévy bridge is assumed to start at time 0 and to end at time 1 with parameters as given in Table 1.

\begin{tabular}{lcccc}
\hline & \multicolumn{2}{c}{ FPT probability } & \multicolumn{2}{c}{ Expected occupation time } \\
\hline$n$ & $P_{n}$ HEJD & $P_{1: n}$ HEJD & $P_{n}$ HEJD & $P_{1: n}$ HEJD \\
\hline 1 & 0.3006853 & 0.3006853 & 0.3680801 & 0.3680801 \\
2 & 0.3617512 & 0.4228170 & 0.4142655 & 0.4604509 \\
3 & 0.3911554 & 0.4635372 & 0.4322124 & 0.4719338 \\
4 & 0.4084846 & 0.4734619 & 0.4415893 & 0.4711338 \\
5 & 0.4198448 & 0.4735378 & 0.4473202 & 0.4707490 \\
6 & 0.4278257 & 0.4720958 & 0.4511786 & 0.4708328 \\
7 & 0.4337174 & 0.4713210 & 0.4539517 & 0.4708704 \\
8 & 0.4382332 & 0.4711443 & 0.4560403 & 0.4708630 \\
9 & 0.4417979 & 0.4711707 & 0.4576699 & 0.4708578 \\
10 & 0.4446794 & 0.4712065 & 0.4589767 & 0.4708575 \\
11 & 0.4470546 & 0.4712177 & 0.4600480 & 0.4708575 \\
\hline
\end{tabular}

Empirically, we observe that the rate of decay of the error of the unextrapolated outcomes to be (approximately) linear for both different functionals, in line with the theoretical error bound given in Corollary 3.1. Indeed, the ordinary least squares (OLS) regression lines (dotted) in the log-log plots had slopes equal to $-0.94(-0.98)$ and $-0.98(-0.99)$ in the case of the first-passage probabilities (and expected occupation times) of the Lévy bridges corresponding to the HEJD model. Moreover, in line with the theoretical error estimates given in Theorem 3.1, we observe that the application of the Richardson extrapolation leads to a significantly faster decay of the error. By comparing the error plots of the expectations of the two path-dependent 
functionals we note that the logarithmic errors for the expected occupation times (for a given $n$ ) are consistently and significantly the smaller of the two, suggesting that the randomisation method converges faster in this case. This feature is likely to be related to the higher degree of smoothness in the case of the expected occupation time. Finally, we mention that we computed the roots of the Cramér-Lundberg equation featuring in the solutions $D_{n, q}$ and $\Omega_{n, q}$ by deploying the Newton-Raphson method.

Remark 4.1. (i) We investigated the round-off error resulting from the computation of the roots based on single precision arithmetic, and found that in that case the computed roots were accurate up to an error of $1.0 \times \mathrm{e}^{-11}$.

(ii) In order to efficiently approximate the first-passage time probability and the expected occupation time of the Lévy bridge process, one could combine the procedure described in this section with interpolation. One would then compute these quantities for a grid of points and subsequently construct functions on the real line $\mathbb{R}$ by using (linear) interpolation.

\section{Illustration: option valuation using the bridge sampling method}

By way of illustration, we present next the numerical results that were obtained by valuing an up-and-in barrier option and a range note under a number of models by using a Markov bridge algorithm described in Algorithm 5.1 below (the recursive method for approximation of first-passage time probabilities and expected occupation times from Section 4 is applied).

We assume that the stock price process $S=\left\{S_{t}, t \in \mathbb{R}_{+}\right\}$evolves according to a Bates-type stochastic volatility model with mixed-exponential jumps. The process $S$ is thus specified by the exponential model $S_{t}=\exp \left\{Y_{t}\right\}, t \in \mathbb{R}_{+}$, where the log-price process $Y=\left\{Y_{t}, t \in \mathbb{R}_{+}\right\}$ satisfies the SDE

$$
\begin{gathered}
\mathrm{d} Y_{t}=\left(\mu-\frac{Z_{t}}{2}\right) \mathrm{d} t+\sqrt{\left|Z_{t}\right|} \mathrm{d} B_{t}+\mathrm{d} J_{t}, \quad Y_{0}=x, \\
\mathrm{~d} Z_{t}=\kappa\left(\delta-Z_{t}\right) \mathrm{d} t+\xi \sqrt{\left|Z_{t}\right|} \mathrm{d} W_{t}, \quad t \in \mathbb{R}_{+}, Z_{0}=v,
\end{gathered}
$$

where $x$ and $v$ are strictly positive, $(B, W)$ is a two-dimensional Brownian motion with correlation parameter $\rho$, and $J_{t}$ is an independent compound Poisson process with intensity $\lambda$ and jump sizes distributed according to a mixed-exponential distribution $F$ with mean $m$. The parameters $\kappa, \delta$, and $\xi$ of the model are positive and represent the speed of mean-reversion of the volatility, the long-term volatility level and the volatility of the volatility parameter. The parameter $\mu$ is set equal to $\mu=r-q-\lambda m$ which ensures that the moment condition $\mathbb{E}\left[\exp \left\{Y_{t}\right\}\right]=\exp \left\{(r-q) t+Y_{0}\right\}$ is satisfied for all nonnegative $t$, where the constants $r$ and $q$ are nonnegative constants representing the risk-free rate of return and the dividend yield. Under this moment condition it holds that the process $\left\{\mathrm{e}^{-(r-q) t} S_{t}, t \in \mathbb{R}_{+}\right\}$is a martingale. Note that choosing $\kappa$ and $\xi$ equal to 0 yields the MEJD process.

By way of example we consider an up-and-in call (UIC) option and a range note (RN). By arbitrage pricing theory, the UIC option and the RN have values at time 0 given by

$$
\begin{gathered}
\operatorname{UIC}(K, H)=\mathbb{E}\left[\mathrm{e}^{-r T}\left(S_{T}-K\right)^{+} \mathbf{1}_{\left\{\sup _{0 \leq t \leq T} S_{t}>H\right\}}\right], \\
\operatorname{RN}\left(a_{1}, a_{2}\right)=\mathbb{E}\left[\mathrm{e}^{-r T} \frac{C}{T} \int_{0}^{T} \mathbf{1}_{\left\{a_{1} \leq S_{u} \leq a_{2}\right\}} \mathrm{d} u\right],
\end{gathered}
$$

where $K$ is the strike price, $H$ is the barrier level, $C$ is the nominal, and $a_{1}$ and $a_{2}$ are the lower and upper bound of the range, respectively. 


\subsection{Markov bridge sampling method}

The first step is to approximate the log-price process $Y$ by a process that has piecewise constant drift and volatility deploying the EM approximation of the process $(Y, Z)$ on the equidistant partition $\mathbb{T}_{N}$, which can be expressed as

$$
\begin{gathered}
Y_{\tau_{n+1}}^{\prime}=Y_{\tau_{n}}^{\prime}+\left(\mu-\frac{Z_{\tau_{n}}^{\prime}}{2}\right) \Delta_{n}+\sqrt{\left|Z_{\tau_{n}}^{\prime}\right| \Delta W_{n}+\Delta J_{n},} \quad Y_{0}^{\prime}=x, \\
Z_{\tau_{n+1}}^{\prime}=Z_{\tau_{n}}^{\prime}+\kappa\left(\delta-Z_{\tau_{n}}^{\prime}\right) \Delta_{n}+\xi \sqrt{\left|Z_{\tau_{n}}^{\prime}\right|} \Delta B_{n}, \quad Z_{0}^{\prime}=v,
\end{gathered}
$$

for $n \in \mathbb{N} \backslash\{0\}$, with $\Delta W_{n}=W_{\tau_{n+1}}-W_{\tau_{n}}, \Delta B_{n}=B_{\tau_{n+1}}-B_{\tau_{n}}, \Delta J_{n}=J_{\tau_{n+1}}-J_{\tau_{n}}$, and $\Delta_{n}=\tau_{n+1}-\tau_{n}=T / N$; see [23] and [27] for results on strong and weak-convergence of this scheme. The Markov bridge-sampling method is based on the continuous-time EM approximation $Y^{\prime}$ leaving the (piecewise constant) approximation $\left(Z_{\tau_{n}}^{\prime}\right)_{n \in \mathbb{N}}$ for $Z$ given in (5.4) unchanged. We arrive at the approximation

$$
Y_{t}^{\prime}=Y_{\tau_{n}}^{\prime}+\left(\mu-\frac{Z_{\tau_{n}}}{2}\right)\left(t-\tau_{n}\right)+\sqrt{\left|Z_{\tau_{n}}^{\prime}\right|}\left(W_{t}-W_{\tau_{n}}\right)+\left(J_{t}-J_{\tau_{n}}\right), \quad Z_{t}^{\prime}=Z_{\tau_{n}}^{\prime}
$$

for $t \in\left[\tau_{n}, \tau_{n+1}\right]$. Observe that with this choice of interpolation it holds that, conditional on the values of the random variable $Z_{\tau_{n}}^{\prime}$, the process $\left\{Y_{t-\tau_{n}}^{\prime}, t \in\left[\tau_{n}, \tau_{n+1}\right]\right\}$ is a Lévy process for each $n=0, \ldots, N-1$. The bridge sampling algorithm is summarised as follows.

Algorithm 5.1. Bridge sampling algorithm for approximating $\mathbb{E}[F(T, Y, Z)]$.

0 . Fix $M, N \in \mathbb{N}$ sufficiently large.

1. Sample $M$ i.i.d. copies $\xi^{(1)}, \ldots, \xi^{(M)}$ from the law of $\left(Y_{\tau_{1}}^{\prime}, Z_{\tau_{1}}^{\prime}, \ldots, Y_{\tau_{N}}^{\prime}, Z_{\tau_{N}}^{\prime}\right)$.

2. Evaluate the estimator $(1 / M) \sum_{i=1}^{M} \widetilde{F}^{(N)}\left(\xi^{(i)}\right)$ with

$$
\begin{aligned}
& \widetilde{F}^{(N)}\left(y_{0}, z_{0}, \ldots, y_{N}, z_{N}\right) \\
& \quad=\mathbb{E}\left[F\left(T, Y^{\prime}, Z^{\prime}\right) \mid Y_{\tau_{0}}^{\prime}=y_{0}, Z_{\tau_{0}}^{\prime}=z_{0}, \ldots, Y_{\tau_{N}}^{\prime}=y_{N}, Z_{\tau_{N}}^{\prime}=z_{N}\right] .
\end{aligned}
$$

Remark 5.1. The choice of $N=1$ in the above algorithm corresponds to the case of a single large step bridge sampling, which is the version of the algorithm that was implemented to produce the results reported in Section 4.

Next we focus on the application of the bridge sampling method to the approximation of the expectation of two path-dependent functionals that are given in terms of the running maximum and the occupation time of $Y$ as

$$
F_{S}(T, Y, Z):=g\left(Y_{T}\right) \mathbf{1}_{\left\{\bar{Y}_{T \leq a\}}\right.}, \quad a>0
$$

with $\bar{Y}_{t}:=\sup \left\{Y_{s}: s \leq t\right\}$,

$$
F_{O}(T, Y, Z):=\int_{0}^{T} g\left(Y_{s}\right) \mathrm{d} s
$$

for some function $g: \mathbb{R}_{+} \rightarrow \mathbb{R}$. The functionals $F_{S}$ and $F_{O}$ admit the following multiplicative 
and additive decompositions into parts that only involve the processes $Y^{i-1, i}:=\left\{Y_{t+\tau_{i-1}}, t \in\right.$ $\left.\left[0, \tau_{i}-\tau_{i-1}\right]\right\}$ for $i=1, \ldots, N$ :

$$
\begin{aligned}
& F_{S}(T, Y, Z)=g\left(Y_{T}\right) \prod_{i=1}^{N} F_{S}^{(i)}(Y, Z), \quad F_{S}^{(i)}(Y, Z)=\mathbf{1}_{\left\{\sup _{s \in\left[\tau_{i-1}, \tau_{i}\right]} Y_{s} \leq a\right\}}, \\
& F_{O}(T, Y, Z)=\sum_{i=1}^{N} F_{O}^{(i)}(Y, Z), \quad F_{O}^{(i)}(Y, Z)=\int_{\tau_{i-1}}^{\tau_{i}} g\left(Y_{s}\right) \mathrm{d} s .
\end{aligned}
$$

These decompositions in turn imply that the conditional expectations

$$
\begin{aligned}
& \widetilde{F}_{S}^{(N)}\left(y_{0}, z_{0}, \ldots, y_{N}, z_{N}\right) \\
& \quad:=\mathbb{E}\left[F_{S}\left(T, Y^{\prime}, Z^{\prime}\right) \mid Y_{\tau_{0}}^{\prime}=y_{0}, Z_{\tau_{0}}^{\prime}=z_{0}, \ldots, Y_{\tau_{N}}^{\prime}=y_{N}, Z_{\tau_{N}}^{\prime}=z_{N}\right], \\
& \widetilde{F}_{O}^{(N)}\left(y_{0}, z_{0}, \ldots, y_{N}, z_{N}\right) \\
& \quad:=\mathbb{E}\left[F_{O}\left(T, Y^{\prime}, Z^{\prime}\right) \mid Y_{\tau_{0}}^{\prime}=y_{0}, Z_{\tau_{0}}^{\prime}=z_{0}, \ldots, Y_{\tau_{N}}^{\prime}=y_{N}, Z_{\tau_{N}}^{\prime}=z_{N}\right]
\end{aligned}
$$

can be expressed in terms of Lévy bridge processes, as shown next.

Proposition 5.1. For any $N \in \mathbb{N}$ the following decompositions hold true:

$$
\begin{gathered}
\widetilde{F}_{S}^{(N)}\left(\left(y_{0}, z_{0}\right), \ldots,\left(y_{N}, z_{N}\right)\right)=g\left(y_{N}\right) \prod_{i=1}^{N} \widetilde{F}_{S}^{(i)}\left(y_{i-1}, y_{i}, z_{i-1}\right), \\
\widetilde{F}_{O}^{(N)}\left(\left(y_{0}, z_{0}\right), \ldots,\left(y_{N}, z_{N}\right)\right)=\sum_{i=1}^{N} \widetilde{F}_{O}^{(i)}\left(y_{i-1}, y_{i}, z_{i-1}\right),
\end{gathered}
$$

where the functions $x \mapsto \widetilde{F}_{S}^{(i)}(x, y, z)$ and $x \mapsto \widetilde{F}_{O}^{(i)}(x, y, z)$ are given by

$$
\widetilde{F}_{S}^{(i)}(x, y, z)=\mathbb{E}\left[\mathbf{1}_{\left(\sup _{s \leq \Delta} L_{s}^{(0, x) \rightarrow(\Delta, y), i} \leq a\right)}\right], \quad \widetilde{F}_{O}^{(i)}(x, y, z)=\mathbb{E}\left[\int_{0}^{\Delta} g\left(L_{s}^{(0, x) \rightarrow(\Delta, y), i}\right) \mathrm{d} s\right]
$$

with $\Delta=T / N$, where $L^{(0, x) \rightarrow(\Delta, y), i}$ denotes the Lévy bridge process from $(0, x)$ to $(\Delta, y)$ with underlying Lévy process $L^{(i)}$ that is equal in law to $Y^{i-1, i}$ conditional on $Z_{\tau_{i-1}}=z$ and $Y_{\tau_{i}}=x$.

Proof. The decompositions hold true as a consequence of the harness property of a Lévy process, the definition of a Lévy bridge, and the fact that a Lévy process is temporally homogeneous.

\subsection{Bates-type stochastic volatility model with jumps}

By approximating the log-price process $Y$ of the Bates-type model by the EM scheme in (5.3)-(5.5), and computing first-passage time probabilities and expected occupation times of the process $Y^{\prime}$ as before using the recursive algorithm (as in Section 4), we obtained the approximate values of an up-and-in call option and a range note under the Heston model and Bates-type models with double-exponential and hyper exponential jumps. We ran Algorithm 5.1 with 10 million paths $\left(M=10^{7}\right)$ on a uniform grid $\Upsilon$ with $N=2^{i}$ steps for $i=0,1, \ldots, 10$. We used the recursions with $n=7$ steps and approximated the functions $\widehat{F}_{S}^{(i)}(x, y, z)$ by evaluating these on a grid of points and using (trilinear) interpolation to obtain approximations of the values of the function outside the grid. By way of comparison, we also report the results obtained by a standard (discrete-time) EM approximation with 10 million paths and a varying number of (equidistant) time-steps. 
TABLE 3: Model parameters of the generalised Bates-type model. The maturity, strike, barrier and spot levels, range of the up-and-in call option, and range note to be used in Figure 2 and Table 4 (with jump parameters as given in Table 1).

\begin{tabular}{cccccccccccc}
\hline$\kappa$ & $\delta$ & $\xi$ & $\rho$ & $V_{0}$ & $K$ & $H$ & $\left(a_{1}, a_{2}\right)$ & $S_{0}$ & $r$ & $d$ & $T$ \\
\hline 1.00 & 0.10 & 0.20 & -0.50 & 0.07 & 100 & 120 & $(1.15,1.35)$ & 100 & 0.05 & 0.00 & 1.00 \\
\hline
\end{tabular}

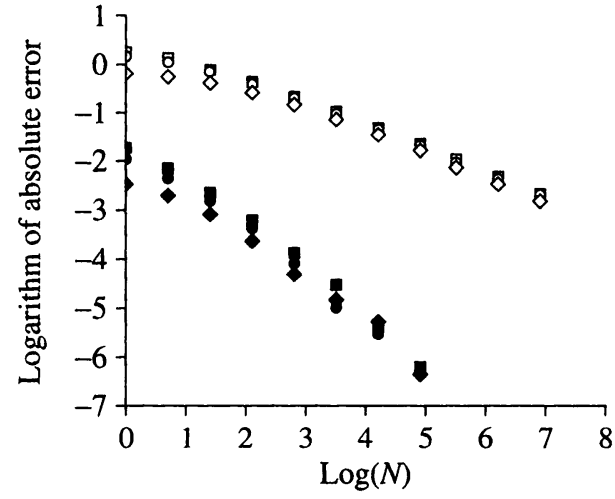

(a) Up-and-in call option

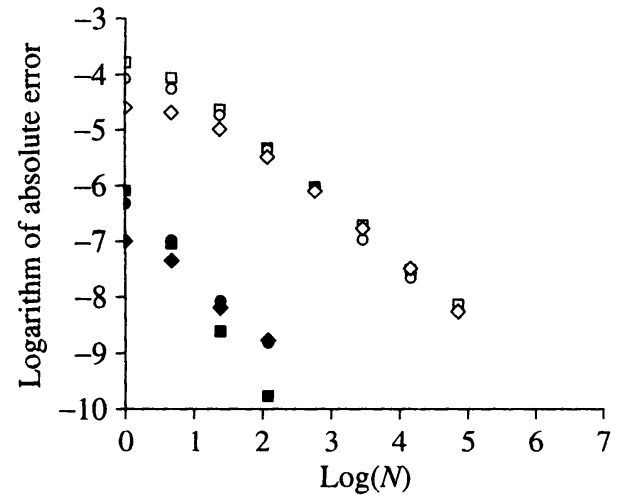

(b) Range note

\begin{tabular}{|lll|}
\hline- Discrete Heston & $\circ$ Discrete Bates (KOU) & $\diamond$ Discrete Bates (HEJD) \\
- Continuous Heston & $\bullet$ Continuous Bates (KOU) & $\bullet$ Continouos Bates (HEJD) \\
\hline
\end{tabular}

FIGURE 2: The absolute error of the values of an up-and-in call option and range note under the Heston and Bates-type models plotted on a log-log scale against the number of time-steps $N$. Parameters are as given in Tables 1 and 3.

For the results shown in Figure 2 we take the value corresponding to $N=1024$ as true value and compute the logarithm of the absolute errors for all other outcomes with respect to this value. In order to estimate the rates of decay of the error we added OLS regression lines to the figures. The slopes of the OLS lines for the Heston model and the Bates-type model with double-exponential and hyper-exponential jumps that we found are $-1.03,-1.02$, and -1.04 in the case of the up-and-in call option and $-1.36,-0.96$, and -1.02 , in the case of the range note, which suggests a rate of decay of the error that is linear in the reciprocal of the number of steps.

By way of comparison we also implemented the standard (discrete-time) EM scheme for each of the three models and found the corresponding three slopes of the OLS lines to be equal to -0.48 in the case of the values of the up-and-in call options and to -1.00 in the case of values of the range notes. These results suggest that, in the case of an UIC option, only a square-root rate holds for the decay of the error as function of the reciprocal of the number of time-steps rather than a linear rate, which is in line with the well-known fact that the strong order of the discrete-time EM scheme is 0.5 , and that, furthermore, for killed diffusion models the weak error of the discrete-time EM scheme has been shown to be bounded by a constant times $N^{-1 / 2}$ in the number of time-steps $N$ under suitable regularity assumptions on the coefficients and the payoff; see [21, Theorems 2.3 and 2.4]. 


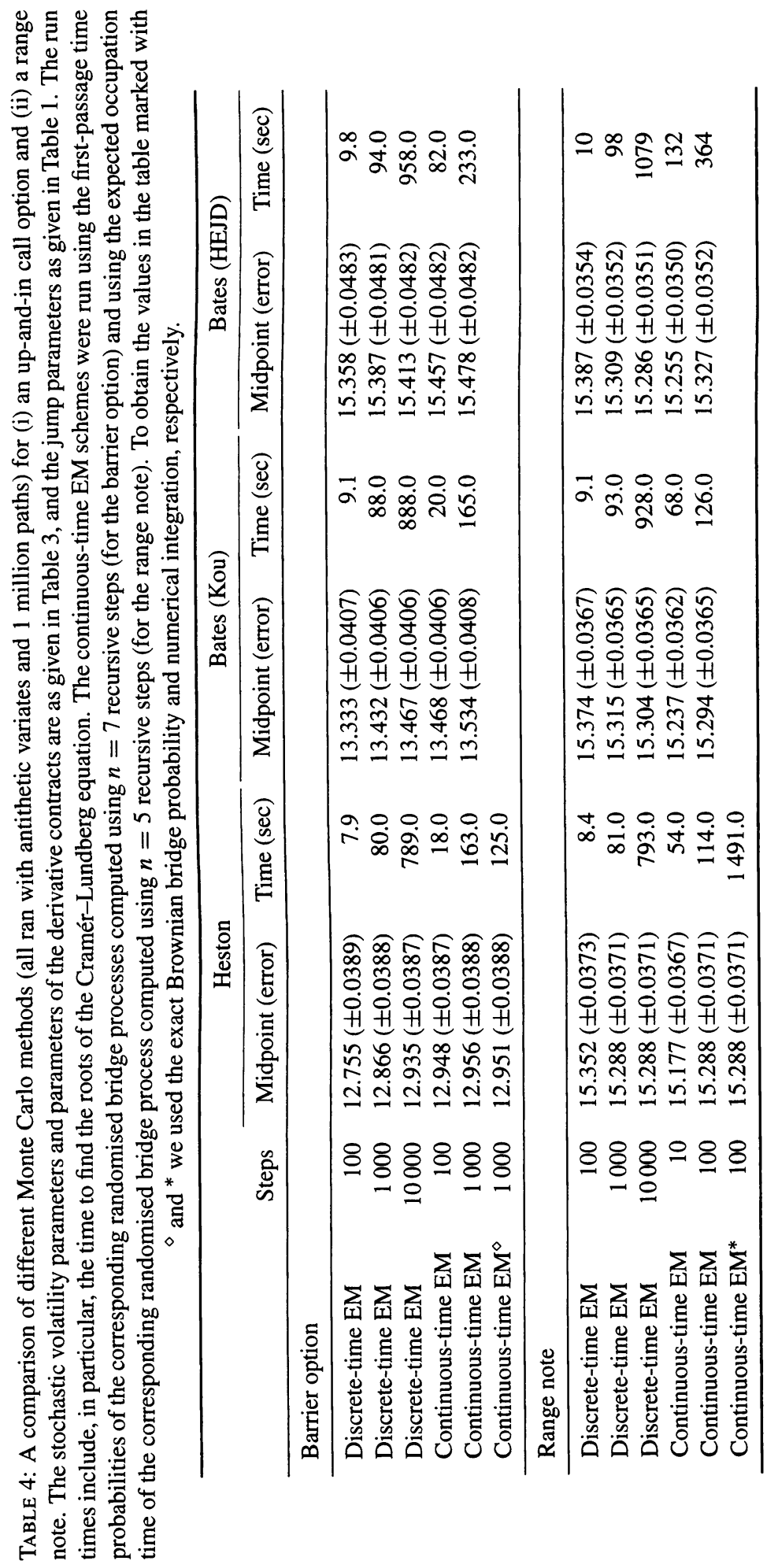




\section{Appendix A. Recursions for maxima and occupation times of Lévy bridges}

Let $X=\left\{X_{t}, t \in \mathbb{R}_{+}\right\}$be a Lévy process (a stochastic process with stationary and independent increments and right-continuous paths with left limits such that $X_{0}=0$ ) that is defined on some filtered probability space $(\Omega, \mathcal{F}, \boldsymbol{F}, \boldsymbol{P})$, where $\boldsymbol{F}=\left\{\mathcal{F}_{t}, t \in \mathbb{R}_{+}\right\}$denotes the completed right-continuous filtration generated by $X$. See [31] and [39] for general treatments of the theory of Lévy processes. To avoid degeneracies we exclude in the sequel the case that $|X|$ is a subordinator. The bridge method under consideration involves randomised bridge processes that can be informally described as processes that are equal in law to $X$ conditioned to take a given value at certain independent random times.

Formally, such a process can be constructed by invoking general results on the existence of conditional distributions and disintegration; see [25, Theorems 6.3 and 6.4]. More specifically, let the triplet $\left(X, \tau_{1}, \tau_{2}\right)$ of the Lévy process $X$ and independent random times $\tau_{1}, \tau_{2}$ with $\tau_{1} \leq \tau_{2}$ be defined on the Borel space $D \times U$ that is the product of the Skorokhod space $D$ of RCLL (right continuous with left limits) functions and the space $U=\mathbb{R}_{+}^{2}$. Then, by disintegration, we obtain a family of conditional laws conditional on different values of $\left(\eta_{1}, \eta_{2}\right):=\left(X_{\tau_{1}}, X_{\tau_{2}}\right)$ that may be used to define the randomised bridge process with starting point $\left(\tau_{1}, y_{1}\right)$ and end point $\left(\tau_{2}, y_{2}\right)$ by $\left\{X_{\left(s+\tau_{1}\right) \wedge \tau_{2}}, s \in \mathbb{R}_{+}\right\}$for almost all realisations $\left(y_{1}, y_{2}\right)$ of $\left(\eta_{1}, \eta_{2}\right)$.

Under regularity assumptions on the Lévy process $X$ and for specific choices of the random times the construction in the previous paragraph may be extended to all realisations of $\left(\eta_{1}, \eta_{2}\right)$, drawing on results in [14] where weak-continuity results and pathwise constructions of a Markov bridges have been recently provided (see also [42] for the case of Lévy processes conditioned to stay positive).

Assumption A.1. The Lévy process $X$ satisfies the integrability condition

$$
\int_{\mathbb{R} \backslash(-1,1)} \frac{\mathrm{d} \theta}{|\Psi(\theta)|}<\infty
$$

where $\Psi$ is the characteristic exponent of $X$, which is the function $\Psi: \mathbb{R} \rightarrow \mathbb{C}$ that satisfies the identity $\mathbb{E}\left[\exp \left(\mathrm{i} \theta X_{t}\right)\right]=\exp (-t \Psi(\theta))$ for all $\theta \in \mathbb{R}$ and $t \in \mathbb{R}_{+}$.

As random times we consider gamma random variables $\Gamma_{n, q}, n \in \mathbb{N}, q>0$, with mean $n / q$ and variance $n / q^{2}$ that are independent of $X$. We suppose that the pair $\left(X, \Gamma_{n, q}\right)$ is defined on the product space $\left(\Omega \times \mathbb{R}_{+}, \mathcal{F} \otimes B\left(\mathbb{R}_{+}\right), \boldsymbol{P} \times P\right)$. To simplify notation we use in the sequel $\mathbb{P}$ to denote the product-measure $\boldsymbol{P} \times \boldsymbol{P}$. It follows from Sato [39, Proposition 28.1] that under Assumption A.1 the distributions under $\mathbb{P}$ of both $X_{\Gamma_{n, q}}$ and $X_{t}, t>0$, admit continuous densities.

Lemma A.1. Let Assumption A.1 hold. (i) Then for any $q>0$ and $n \in \mathbb{N}$ the random variable $X_{\Gamma_{n, q}}$ has a continuous and bounded density $u_{n, q}$.

(ii) $X_{t}$ has a bounded density $p(t, x)$ that is continuous in $(t, x) \in(0, \infty) \times \mathbb{R}$.

Under Assumption A.1 we may define the randomised Lévy bridge process starting at $(0, x)$ and pinned down at $\left(\Gamma_{n, q}, y\right)$ for any $x, y \in \mathbb{R}$. We recall first from [14, Theorem 1] that, under Assumption A.1 and for any $t>0$ and $x, y \in \mathbb{R}$ such that $p(t, y-$ $x)>0$, there exists a Markov process on the probability space $(\Omega, \mathcal{F}, P)$, denoted by $X^{(0, x) \rightarrow(t, y)}=\left\{X_{u}^{(0, x) \rightarrow(t, y)}, u \in[0, t]\right\}$, that starts at time 0 at $x$ almost surely is equal to $y$ at time $t$ almost surely and satisfies the disintegration property. The process $X^{(0, x) \rightarrow(t, y)}=$ $\left\{X_{u}^{(0, x) \rightarrow(t, y)}, u \in[0, t]\right\}$ is referred to as the Lévy bridge process from $(0,0)$ to $(t, y)$. 
We next specify the definition of a Lévy bridge process pinned down at a gamma random time and a given fixed end point. For any pair $x, y \in \mathbb{R}$ with $u_{n, q}(y-x)>0$, the randomised Lévy bridge process $X^{(0, x) \rightarrow\left(\Gamma_{n, q}, y\right)}=\left\{X_{t}^{(0, x) \rightarrow\left(\Gamma_{n, q}, y\right)}, t \in \mathbb{R}_{+}\right\}$starting from $(0, x)$ and pinned down at $\left(\Gamma_{n, q}, y\right)$ is the stochastic process with sample paths $\left.t \mapsto X_{t \wedge s}^{(0, x) \rightarrow(s, y)}(\omega)\right|_{s=\Gamma_{n, q}(\gamma)}$ for given realisations $(\omega, \gamma)$ in the sample space $\Omega \times \mathbb{R}_{+}$. The process $X^{(0, x) \rightarrow\left(\Gamma_{n, q}, y\right)}$ satisfies the disintegration property (which can be shown by a similar line of reasoning as was given in the proof of [14, Theorem 1]), and is, hence, equal in law to the corresponding process obtained by the construction described in the second paragraph of this section. The derivation of the expressions for the functions $\vec{D}_{q}^{(1)}(x, y)$ and $\vec{\Omega}_{q}^{(1)}(x, y)$ is based in part on the following auxiliary result concerning the differentiability of two related functions under Assumption A.1 (the proof of which is omitted as it follows by standard arguments).

Lemma A.2. Let Assumption A.1 hold and let $q$ be any strictly positive number.

(i) For any fixed $x \in \mathbb{R}_{+}$, the function $y \mapsto \mathbb{P}\left(\bar{X}_{\Gamma_{1, q}} \leq x, X_{\Gamma_{1, q}} \leq y\right)$ is continuously differentiable on $\mathbb{R}$ and its derivative $y \mapsto D_{1, q}(x, y)$ is bounded.

(ii) The map $(x, y) \mapsto \mathbb{E}\left[\int_{0}^{\Gamma_{1, q}} \mathbf{1}_{\left\{X_{u} \leq x\right\}} \mathrm{d} u \mathbf{1}_{\left\{X_{\left.\Gamma_{1, q} \leq y\right\}}\right]}\right.$ is continuously differentiable with respect to $x$ and $y$ in $\mathbb{R}$. The mixed derivative with respect to $x$ and $y$ is given by $\Omega_{1, q}(x, y)$ for $x, y \in \mathbb{R}$.

The functions $D_{1, q}$ and $\Omega_{1, q}$ admit semianalytical expressions, which can be derived using the Markov property and the Wiener-Hopf factorisation of $X$. We recall (see, for example, [7, Chapter VI]) that the probabilistic form of the Wiener-Hopf factorisation of $X$ states that

(a) the running supremum $\bar{X}_{\Gamma_{1, q}}$ and the drawdown $\bar{X}_{\Gamma_{1, q}}-X_{\Gamma_{1, q}}$ of $X$ at the random time $\Gamma_{1, q}$ are independent, and

(b) the drawdown $\bar{X}_{\Gamma_{1, q}}-X_{\Gamma_{1, q}}$ has the same law as the negative of the running infimum $-\underline{X}_{\Gamma_{1, q}}$.

The probabilistic form of the Wiener-Hopf factorisation implies that the characteristic function of the random variable $X_{\Gamma_{1, q}}$ is equal to the product of the characteristic functions $\Psi_{q}^{+}$and $\Psi_{q}^{-}$ of $\bar{X}_{\Gamma_{1, q}}$ and $\underline{X}_{\Gamma_{1, q}}$,

$$
\Psi_{q}^{+}(\theta)=\mathbb{E}\left[\exp \left(\mathrm{i} \theta \bar{X}_{\Gamma_{1, q}}\right)\right], \quad \Psi_{q}^{-}(\theta)=\mathbb{E}\left[\exp \left(\mathrm{i} \theta \underline{X}_{\Gamma_{1, q}}\right)\right]
$$

In the following result we establish that the functions $D_{n, q}, \Omega_{n, q}$ are well defined and satisfy (1.2) and (1.3).

Theorem A.1. Let $q>0, n \in \mathbb{N}$, and let Assumption A.1 hold.

(i) For any $x \in \mathbb{R}_{+}$, the function $y \mapsto \mathbb{P}\left(\bar{X}_{\Gamma_{n, q}} \leq x, X_{\Gamma_{n, q}} \leq y\right)$ admits a continuous bounded density denoted by $D_{n, q}$, and

$$
(x, y) \mapsto \mathbb{E}\left[\int_{0}^{\Gamma_{n, q}} \mathbf{1}_{\left\{X_{u} \leq x\right\}} \mathrm{d} u \mathbf{1}_{\left\{X_{\Gamma_{n, q}} \leq y\right\}}\right]
$$

is continuously differentiable on $\mathbb{R}^{2}$ with bounded mixed-derivative denoted by $\Omega_{n, q}$.

(ii) The functions $D_{n, q}$ and $\Omega_{n, q}$ satisfy (1.2) and (1.3). 
Remark A.1. Since the pinned process $X^{(0,0) \rightarrow\left(\Gamma_{n, q}, y\right)}$ is equal in law to the process $X^{\Gamma_{n, q}}=$ $\left\{X_{u}, u \in\left[0, \Gamma_{n, q}\right]\right\}$ stopped at the random time $\Gamma_{n, q}$ and conditioned on $\left\{X_{\Gamma_{n, q}}=y\right\}$, it follows that the functions $\vec{D}_{q}^{(n)}\left(\vec{\Omega}_{q}^{(n)}\right)$ are equal to the ratio of $D_{n, q}\left(\Omega_{n, q}\right.$, respectively) and $u_{n, q}$, that is, for $x \in \mathbb{R}_{+}$and $y \in \mathbb{R}$,

$$
D_{n, q}(x, y)=\vec{D}_{q}^{(n)}(x, y) u_{n, q}(y), \quad \Omega_{n, q}(x, y)=\frac{\mathrm{d}}{\mathrm{d} x} \vec{\Omega}_{q}^{(n)}(x, y) u_{n, q}(y) .
$$

Proof of Theorem A.1. (i) Several applications of the strong Markov property of $X$ and the lack of memory property of the exponential distribution yield

$$
\begin{aligned}
\mathbb{P}\left[\bar{X}_{\Gamma_{n, q}} \leq x, X_{\Gamma_{n, q}} \in \mathrm{d} y\right] \\
=\mathbb{P}\left[\tau_{x}^{+} \geq \Gamma_{n, q}, X_{\Gamma_{n, q}} \in \mathrm{d} y\right] \\
=\mathbb{P}\left[X_{\Gamma_{n, q}} \in \mathrm{d} y\right]-\sum_{k=1}^{n} \mathbb{P}\left[\Gamma_{k-1, q} \leq \tau_{x}^{+}<\Gamma_{k, q}, X_{\Gamma_{n, q}} \in \mathrm{d} y\right] \\
=\mathbb{P}\left[X_{\Gamma_{n, q}} \in \mathrm{d} y\right] \\
\quad-\sum_{k=1}^{n} \int_{\mathbb{R}_{+}} \mathbb{E}\left[\mathbf{1}_{\left\{\Gamma_{k-1, q} \leq \tau_{x}^{+}<\Gamma_{k, q}\right\}} \mathbf{1}_{\left\{X_{\tau_{x}^{+}} \in \mathrm{d} z\right\}}\right] \mathbb{P}\left[z+X_{\Gamma_{n-k+1, q}} \in \mathrm{d} y\right]
\end{aligned}
$$

with $\Gamma_{0, q}:=0$. The Fourier transform $\mathcal{F} r_{x}(s), s \in \mathbb{R}_{+}$, of the measure $r_{x}^{n, q}$ given by $r_{x}^{n, q}(\mathrm{~d} y):=\mathbb{P}\left[\bar{X}_{\Gamma_{n, q}} \leq x, X_{\Gamma_{n, q}} \in \mathrm{d} y\right]$ can be expressed as

$$
\mathbb{E}\left[\exp \left\{\text { is } X_{\Gamma_{n, q}}\right\}\right]-\sum_{k=1}^{n} \mathbb{E}\left[\exp \left\{\text { is } X_{\Gamma_{n-k+1, q}}\right\}\right] \mathbb{E}\left[\exp \left\{\text { is } X_{\tau_{x}^{+}}\right\} \mathbf{1}_{\left\{\Gamma_{k-1, q} \leq \tau_{x}^{+}<\Gamma_{k, q}\right\}}\right] .
$$

Since the second factors in the sum in the previous display are bounded by 1 and

$$
\mathbb{E}\left[\exp \left(\mathrm{i} \theta X_{\Gamma_{n, q}}\right)\right]=\left(\frac{q}{q+\Psi(\theta)}\right)^{n},
$$

we have $\left|\mathcal{F} r_{x}(s)\right| \leq \sum_{k=1}^{n} \int q^{k}|q+\Psi(s)|^{-k}$ d $s$ for any $x \in \mathbb{R}_{+}, q>0$ and $n \in \mathbb{N}$, which is finite by Assumption A.1 and the bound $|q /(q+\Psi(s))| \leq 1$ that holds for all $s \in \mathbb{R}$. We conclude that, for any $x \in \mathbb{R}_{+}$, the measure $r_{x}^{n, q}(\mathrm{~d} y)$ admits a continuous bounded density (by [39, Proposition 28.1]).

We show the required differentiability of $\mathbb{E}\left[\int_{0}^{\Gamma_{n, q}} \mathbf{1}_{\left\{X_{u} \leq x\right\}} \mathrm{d} u \mathbf{1}_{\left\{X_{\left.\Gamma_{n, q} \leq y\right\}}\right]}\right.$ by induction with respect to $n$. Noting that the $n=1$ case follows from Lemma A.2(ii), we next turn to the induction step. Assume thus that the assertion is valid for given $n \in \mathbb{N}$. By an application of the Markov property, we have

$$
\begin{aligned}
\mathbb{E}\left[\int_{0}^{t+u} \mathbf{1}_{\left\{X_{s} \leq x\right\}} \mathrm{d} s \mathbf{1}_{\left\{X_{t+u} \in \mathrm{d} b\right\}}\right]= & \int_{w \in \mathbb{R}} \mathbb{E}\left[\int_{0}^{t} \mathbf{1}_{\left\{X_{s} \leq x\right\}} \mathrm{d} s \mathbf{1}_{\left\{X_{t} \in \mathrm{d} w\right\}}\right] \mathbb{P}\left[w+X_{u} \in \mathrm{d} b\right] \\
& +\int_{w \in \mathbb{R}} \mathbb{E}\left[\int_{0}^{u} \mathbf{1}_{\left\{w+X_{s} \leq x\right\}} \mathrm{d} s \mathbf{1}_{\left\{w+X_{u} \in \mathrm{d} b\right\}}\right] \mathbb{P}\left[X_{t} \in \mathrm{d} w\right]
\end{aligned}
$$

for any real $x$. Replacing $t$ and $u$ in (A.1) by the independent random times $\Gamma_{1, q}$ and $\Gamma_{n-1, q}$, using the fact that their sum is equal in distribution to $\Gamma_{n, q}$ and that the random variables $X_{\Gamma_{n, q}}$ and $X_{\Gamma_{1, q}}$ have continuous densities $u_{n, q}$ and $u_{1, q}$ (by Lemma A.2), it follows from the induction assumption that the assertion holds for $n+1$. It follows thus by induction that we have the required differentiability for all $n \in \mathbb{N}$. 
(ii) Since we may write

$$
\bar{X}_{t}=\max \left\{X_{s}+\sup _{0 \leq u \leq t-s}\left(X_{u+s}-X_{s}\right), \bar{X}_{s}\right\} \quad \text { for any } s, t
$$

with $0 \leq s \leq t$, it follows as a consequence of the stationarity and independence of increments of $X$, and the fact that a $\Gamma_{n, q}$ random variable is equal in distribution to the sum of independent $\Gamma_{n-1, q}$ and $\Gamma_{1, q}$ random variables, that we have

$$
\begin{aligned}
& \mathbb{P}\left(\bar{X}_{\Gamma_{n, q}} \leq x, X_{\Gamma_{n, q}} \in \mathrm{d} w\right) \\
& \quad=\mathbb{P}\left(\max \left\{X_{\Gamma_{1, q}}+\bar{X}_{\Gamma_{n-1, q}}^{\prime}, \bar{X}_{\Gamma_{1, q}}\right\} \leq x, X_{\Gamma_{1, q}}+X_{\Gamma_{n-1, q}}^{\prime} \in \mathrm{d} w\right) \\
& \quad=\int_{(-\infty, x]} \mathbb{P}\left(\bar{X}_{\Gamma_{1, q}} \leq x, X_{\Gamma_{1, q}} \in \mathrm{d} z\right) \mathbb{P}\left(z+\bar{X}_{\Gamma_{n-1, q}} \leq x, z+X_{\Gamma_{n-1, q}} \in \mathrm{d} w\right),
\end{aligned}
$$

where the random variables $\bar{X}_{\Gamma_{n-1, q}}^{\prime}$ and $X_{\Gamma_{n-1, q}}^{\prime}$ are independent of $X$. We arrive at (1.2) since the Lévy process $X$ is spatially homogeneous.

The recursion follows from (A.1) replacing as before $t$ and $u$ by the independent random times $\Gamma_{1, q}$ and $\Gamma_{n-1, q}$ and using the fact that their sum is equal in distribution to a $\Gamma_{n, q}$ random variable.

\section{Acknowledgements}

We thank the Editor and the anonymous referee. We also thank Dan Crisan, Lane Hughston, Antoine Jacquier, Felicity Pearce, Vladimir Piterbarg, Johannes Ruf, David Taylor, and Josef Teichmann, in addition to the participants of the Global Derivatives Trading \& Risk Management 2012 (Barcelona), the Seventh World Congress of the Bachelier Finance Society 2012 (Sydney), and a satellite workshop at the University of the Witwatersrand 2011 (Johannesburg), for useful comments. JS was supported by an EPSRC DTA grant and a doctoral grant from the German Academic Exchange Service (grant No. D/11/42213).

\section{References}

[1] Asmussen, S. And Albrecher, H. (2010). Ruin Probabilities, 2nd edn. World Scientific, Hackensack, NJ.

[2] Asmussen, S. AND Rosiński, J. (2001). Approximations of small jumps of Lévy processes with a view towards simulation. J. Appl. Prob. 38, 482-493.

[3] Asmussen, S., Avram, F. and Usa bel, M. (2002). Erlangian approximations of finite-horizon ruin probabilities. ASTIN Bull. 32, 267-281.

[4] Avram, F., Chan, T. and Usabel, M. (2002). On the valuation of constant barrier options under spectrally one-sided exponential Lévy models and Carr's approximation for American puts. Stoch. Process. Appl. 100, 75-107.

[5] Bartholomew, D. J. (1969). Sufficient conditions for a mixture of exponentials to be a probability density function. Ann. Math. Statist. 40, 2183-2188.

[6] BATES, D. S. (1996). Jumps and stochastic volatility: exchange rate processes implicit in Deutsche Mark options. Rev. Financial Studies 9, 69-107.

[7] Bertoin, J. (1996). Lévy Processes. Cambridge University Press.

[8] Beskos, A., Papaspiliopoulos, O. and Roberts, G. O. (2006). Retrospective exact simulation of diffusion sample paths with applications. Bernoulli 12, 1077-1098.

[9] BotTA, R. F. AND HARRIS, C. M. (1986). Approximation with generalized hyperexponential distributions: weak convergence results. Queueing Systems 1, 169-190.

[10] BOYARCHENKo, M. AND LEVENDORSKIĬ, S. (2012). Valuation of continuously monitored double barrier options and related securities. Math. Finance 22, 419-444.

[11] Boyle, P., Broadie, M. and Glasserman, P. (1997). Monte Carlo methods for security pricing. J. Econom. Dynam. Control 21, 1267-1321. 
[12] CaI, N. And Kou, S. G. (2011). Option pricing under a mixed-exponential jump diffusion model. Manag. Sci. 57, 2067-2081.

[13] CARR, P. (1998). Randomization and the American put. Rev. Financial Studies 11, 597-626.

[14] Chaumont, L. AND Uribe Bravo, G. (2011). Markovian bridges: weak continuity and pathwise constructions. Ann. Prob. 39, 609-647.

[15] Cont, R. and Tankov, P. (2004). Financial Modelling with Jump Processes. Chapman \& Hall/CRC, Boca Raton, FL.

[16] Dereich, S. (2011). Multilevel Monte Carlo algorithms for Lévy-driven SDEs with Gaussian correction. Ann. Appl. Prob. 21, 283-311.

[17] Feller, W. (1966). An Introduction to Probability Theory and Its Applications, Vol. II. John Wiley, New York.

[18] Ferreiro-Castilla, A., Kyprianou, A. E., Scheichl, R. and Suryanarayana, G. (2014). Multilevel Monte Carlo simulation for Lévy processes based on the Wiener-Hopf factorisation. Stoch. Process. Appl. 124, 985-1010.

[19] Figueroa-LóPEz, J. E. AND TANKov, P. (2014). Small-time asymptotics of stopped Lévy bridges and simulation schemes with controlled bias. Bernoulli 20, 1126-1164.

[20] Gatheral, J. (2006). The Volatility Surface: A Practitioner's Guide. John Wiley, Hoboken, NJ.

[21] Gobet, E. (2000). Weak approximation of killed diffusion using Euler schemes. Stoch. Process. Appl. 87, 167-197.

[22] Haug, E. G. (2007). The Complete Guide to Option Pricing Formulas, 2nd edn. McGraw-Hill, New York.

[23] Higham, D. J. AND MaO, X. (2005). Convergence of Monte Carlo simulations involving the mean-reverting square root process. J. Comp. Finance 8, 35-62.

[24] JEAnnin, M. AND PISToRius, M. (2010). A transform approach to compute prices and Greeks of barrier options driven by a class of Lévy processes. Quant. Finance 10, 629-644.

[25] Kallenberg, O. (2002). Foundations of Modern Probability, 2nd edn. Springer, New York.

[26] Kleinert, F. AND VAN Schaik, K. (2015). A variation of the Canadisation algorithm for the pricing of American options driven by Lévy processes. Stoch. Process. Appl. 125, 3234-3254.

[27] Kloeden, P. AND Neuenkirch, A. (2013). Convergence of numerical methods for stochastic differential equations in mathematical finance. In Recent Developments in Computational Finance, World Scientific, Hackensack, NJ, pp. 49-80.

[28] Kloeden, P. E. ANd Platen, E. (1992). Numerical Solution of Stochastic Differential Equations. Springer, Berlin.

[29] KoU, S. G. AND WANG, H. (2004). Option pricing under a double exponential jump diffusion model. Manag. Sci. 50, 1178-1192.

[30] Kuznetsov, A., Kyprianou, A. E., Pardo, J. C. and van Schaik, K. (2011). A Wiener-Hopf Monte Carlo simulation technique for Lévy processes. Ann. Appl. Prob. 21, 2171-2190.

[31] Kyprianou, A. E. (2006). Introductory Lectures on Fluctuations of Lévy Processes with Applications. Springer, Berlin.

[32] Kyprianou, A. E. and Pistorius, M. R. (2003). Perpetual options and Canadization through fluctuation theory. Ann. Appl. Prob. 13, 1077-1098.

[33] LEVENDORSKIĬ, S. (2011). Convergence of price and sensitivities in Carr's randomization approximation globally and near barrier. SIAM J. Financial Math. 2, 79-111.

[34] LEWIS, A. L. AND MORDECKI, E. (2008). Wiener-Hopf factorization for Lévy processes having positive jumps with rational transforms. J. Appl. Prob. 45, 118-134.

[35] Marchuk, G. I. ANd ShaĬdurov, V. V. (1983). Difference Methods and Their Extrapolations. Springer, New York.

[36] Metwally, S. A. K. AND Atiya, A. F. (2002). Using Brownian bridge for fast simulation of jump-diffusion processes and barrier options. J. Derivatives 10, 43-54.

[37] Press, W. H., Teukolsky, S. A., Vetterling, W. T. and Flannery, B. P. (2002). Numerical Recipes in $C++$, 2nd edn. Cambridge University Press.

[38] Ruf, J. AND Scherer, M. (2011). Pricing corporate bonds in an arbitrary jump-diffusion model based on an improved Brownian-bridge algorithm. J. Comp. Finance 14, 127-145.

[39] Sato, K.-I. (1999). Lévy Processes and Infinitely Divisible Distributions. Cambridge University Press.

[40] Sidi, A. (2003). Practical Extrapolation Methods: Theory and Applications. Cambridge University Press.

[41] Stolte, J. (2013). On accurate and efficient valuation of financial contracts under models with jumps. Doctoral Thesis, Imperial College London.

[42] Uribe Bravo, G. (2014). Bridges of Lévy processes conditioned to stay positive. Bernoulli 20, 190-206. 